\title{
Differential PilA pilus assembly by a hospital-acquired and a community-derived Enterococcus faecium isolate
}

Correspondence

Antoni P. A. Hendrickx

ahendric@bsd.uchicago.edu

Received 09 May 2010

Accepted 09 June 2010

\author{
Antoni P. A. Hendrickx ${ }^{1}{ }^{\dagger}$ Claudia M. E. Schapendonk, ${ }^{1}$ \\ Miranda van Luit-Asbroek, ${ }^{1}$ Marc J. M. Bonten, ${ }^{1,2}$ Willem van Schaik ${ }^{1}$ \\ and Rob J. L. Willems ${ }^{1}$
${ }^{1}$ Department of Medical Microbiology, University Medical Center Utrecht, Utrecht, The Netherlands
2 Julius Center for Health Sciences and Primary Care, University Medical Center Utrecht, Utrecht, The Netherlands

\begin{abstract}
Pili are hair-like structures protruding from the cell envelope of bacterial cells. Here, we describe the conditional and differential display of PilA-type pili, and PilE and PilF proteins, encoded from pilin gene cluster 1 at the surface of a hospital-acquired Enterococcus faecium bloodstream isolate (E1165) and a community-derived stool isolate (E1039), at two different temperatures. Both strains have virtually identical pilA gene clusters, as determined by sequencing. Western blotting and transmission immunoelectron microscopy revealed that PilA and PilF assembled into high-molecular-mass pilus-like structures at $37{ }^{\circ} \mathrm{C}$ in the $\mathrm{E} 1165$ strain, whereas PilE was not produced at either of the temperatures used; at $21^{\circ} \mathrm{C}$, PilA and PilF were cell-wall-anchored proteins. In contrast, in strain E1039, PilA, PilE and PilF pilin proteins were found to be displayed as cell-wall-anchored proteins at $37{ }^{\circ} \mathrm{C}$ only, and they were not associated with pilus-like structures. The discrepancy in pilus assembly between E1039 and E1165 cannot be explained by differences in expression of the genes encoding the predicted sortases in the pilA gene cluster, as these had similar expression levels in both strains at 21 and $37{ }^{\circ} \mathrm{C}$. Double-labelling electron microscopy revealed that PilA formed the pilus backbone in E1165, and PilF the minor subunit which was distributed along the PilA pilus shaft and positioned at the tip; however, it was deposited as a cell-wall-anchored protein in a pilA isogenic mutant. The differential deposition of surface proteins from pilin gene cluster 1 and differences in pilus assembly in the two strains suggest a complex post-transcriptional regulatory mechanism of pilus biogenesis in $E$. faecium.
\end{abstract}

\section{INTRODUCTION}

Enterococci are antimicrobial-resistant opportunistic pathogens that are increasingly causing healthcare-associated infections (Murray, 2000; Werner et al., 2008). Both Enterococcus faecium and Enterococcus faecalis have rapidly emerged in hospitals around the world, and they are responsible for infections in the immunocompromised host (Hidron et al., 2008; Murdoch et al., 2002). Today, enterococci rank third among nosocomial pathogens in the USA, as reported by the National Healthcare Safety Network (Hidron et al., 2008). Hospital-acquired E. faecium isolates associated with hospital

†Present address: Department of Microbiology, University of Chicago, Chicago, IL 60637, USA.

Abbreviations: $\mathrm{BHI}$, brain heart infusion; CWS, cell wall sorting signal; FACS, fluorescence-activated cell sorting; HSA, human serum albumin; MLST, multilocus sequence typing; MSCRAMM, microbial surface component recognizing adhesive matrix molecule; PBSb, 1\% BSA in PBS; PGC, pilin gene cluster; TEM, transmission immunoelectron microscopy; TSA, trypticase soy agar II. outbreaks and invasive infections frequently show high-level resistance to ampicillin and ciprofloxacin, and can be distinguished from community-derived isolates based on their gene content (Leavis et al., 2006, 2007; Willems et al., 2005). Hospital-acquired E. faecium isolates are enriched in genes encoding LPXTG-like cell-wall-anchored surface proteins (Hendrickx et al., 2007), including the gene encoding the enterococcal surface protein Esp (Leavis et al., 2004), a collagen- and fibrinogen-binding microbial surface component recognizing adhesive matrix molecule (MSCRAMM) called EcbA, a nidogen and fibrinogen adhesin designated SgrA, and several LPXTG surface protein genes located near one or more predicted sortase genes in pilin gene clusters (PGCs) (Hendrickx et al., 2008, 2009a). Recent in silico analysis has revealed the presence of four PGCs in E. faecium, and specific sera directed against two putative major pilin subunits, which are designated PilA and PilB and are encoded from PGC-1 and -3 , respectively, have been shown to react with two phenotypically distinct pilus-like structures on the surface of a clinical E. faecium isolate (Hendrickx et al., 2008). 
Pili, also referred to as fimbriae, are hair-like proteinaceous filaments protruding from the bacterial cell wall, and they are implicated in virulence of several Gram-positive pathogens, including E. faecalis (Abbot et al., 2007; Dramsi et al., 2006b; Gaspar \& Ton-That, 2006; Nallapareddy et al., 2006b; Nelson et al., 2007). In contrast with Gram-negative organisms, pili of Gram-positive organisms are covalently joined polymers that are tightly immobilized to the peptidoglycan wall (Marraffini \& Schneewind, 2006; TonThat \& Schneewind, 2003). The prototype heterotrimeric pilus is composed of a major subunit that forms the pilus backbone, a minor pilin subunit distributed along the pilus shaft, and an adhesive tip protein. The putative E. faecium major pilin subunits contain an $\mathrm{N}$-terminal signal sequence for initiation of Sec-mediated translocation across the plasma membrane, a HLYPKN pilin motif, an ETXAPExY E box motif, and a C-terminal cell wall sorting signal (CWS). The CWS comprises an LPXTG-like sortase substrate sequence, and a hydrophobic domain, followed by positively charged amino acids (Schneewind et al., 1992, 1993; Ton-That \& Schneewind, 2003). Conservation of these sequence elements in putative major pilin subunits of E. faecium, and in pilin subunits from other Gram-positive bacteria, suggests a universal mechanism of pilus assembly that may also be applied to enterococci (Hendrickx et al., 2009b).

To gain insight into pilus assembly of E. faecium, we studied pilin protein display and PilA pilus biogenesis at the surfaces of a clinical wound isolate (E1165), an isogenic pilA mutant of E1165 and a community-derived human stool isolate (E1039), at different growth temperatures.

\section{METHODS}

Bacterial strains and growth conditions. The bacterial strains and plasmids used in this study are listed in Table 1. E. faecium strains E1162 and E1165 are clinical isolates harvested from bloodstream and wound infections, respectively, whereas E1360 and E1391 are clinical isolates with an undetermined isolation site. Strains E128, E1039 and E1133 are faecal isolates from healthy human volunteers. All strains have been typed previously by our group using multilocus sequence typing (MLST) (Willems et al., 2005). E. faecium strains were grown aerobically at 21 or $37{ }^{\circ} \mathrm{C}$ on trypticase soy agar II (TSA) supplemented with $5 \%$ sheep blood (Becton Dickinson), or in brain heart infusion (BHI) broth, at $37^{\circ} \mathrm{C}$, unless stated otherwise. When appropriate, chloramphenicol and gentamicin (Sigma-Aldrich) were used at concentrations of $15-34$ and $125 \mu \mathrm{g} \mathrm{ml}{ }^{-1}$, respectively. A temperature-sensitive vector (pTEX5500ts) was used to introduce an insertion-deletion mutation in the pilA gene of E. faecium E1165. The oligonucleotides used in this study are listed in Table 2.

\begin{abstract}
Identification of PGC-1 in E. faecium isolates. Dot-blot analysis was performed on chromosomal DNA isolated from E. faecium E128, E1039, E1133, E1162, E1360 and E1391 to determine the presence of the pilA and pilF genes from PGC-1. Chromosomal DNA isolation was performed as described previously (Hendrickx et al., 2007). The chromosomal DNA was denatured with $0.5 \mathrm{M} \mathrm{NaOH}$ in a 96-well microtitre plate for $15 \mathrm{~min}$ and then transferred to a Bio-Rad Bio dot apparatus, vacuum blotted onto an Hybond- $\mathrm{N}^{+}$nylon membrane and fixed onto the membrane by incubation for $2 \mathrm{~min}$ in $0.4 \mathrm{M} \mathrm{NaOH}$, followed by neutralization in $10 \times$ SSC for $1 \mathrm{~min}$. Membranes were hybridized overnight at $42{ }^{\circ} \mathrm{C}$ with a $100 \mathrm{ng}$ probe. Gene-specific probes were amplified by PCR using primer pairs specific for pilA and pilF (Table 2). Amplified DNA probes were purified with a PCR purification kit (Qiagen) and labelled according to the ECL nucleic acid labelling kit (GE Healthcare). The membranes were exposed to Hyperfilm ECL (GE Healthcare).
\end{abstract}

Antipeptide polyclonal rabbit antisera. Specific polyclonal rabbit antisera were prepared by Eurogentec, according to their 87 day

Table 1. Bacterial strains and plasmids used in this study

NA, Not applicable; ts, temperature sensitive; ST, sequence type.

\begin{tabular}{|c|c|c|c|}
\hline Strain or plasmic & Relevant characteristics & ST & Reference \\
\hline \multicolumn{4}{|l|}{ Strains } \\
\hline E. coli $\mathrm{DH} 5 \alpha$ & E. coli host strain for routine cloning & NA & Hanahan (1983) \\
\hline \multicolumn{4}{|l|}{ E. faecium } \\
\hline E128 & Community surveillance isolate from faeces, pilA and pilF negative & 82 & This study \\
\hline E1039 & Community surveillance isolate from faeces, pilA and pilF positive & 42 & This study \\
\hline E1133 & Community surveillance isolate from faeces, pilA and pilF positive & 117 & This study \\
\hline E1162 & Clinical blood isolate, pilA and pilF positive, $\mathrm{Cm}^{\mathrm{S}} \mathrm{Gm}^{\mathrm{s}}$ & 17 & Heikens et al. (2007) \\
\hline E1165 & Clinical wound isolate, pilA and pilF positive, intermediate $\mathrm{Cm}^{\mathrm{R}} \mathrm{Gm}^{\mathrm{s}}$ & 17 & Hendrickx et al. (2008) \\
\hline E1165 $\Delta$ pilA & E1165 with a disrupted pilA gene, pilF positive $\mathrm{Cm}^{\mathrm{R}} \mathrm{Gm}^{\mathrm{S}}$ & 17 & This study \\
\hline E1360 & Clinical isolate, undetermined isolation site, pilA and pilF positive & 16 & This study \\
\hline E1391 & Clinical isolate, undetermined isolation site, pilA and pilF positive & 16 & This study \\
\hline E1674 & Surveillance isolate from faeces, pilA and pilF positive & 110 & This study \\
\hline \multicolumn{4}{|l|}{ Plasmids } \\
\hline pTEX5500ts & Shuttle plasmid, ts in Gram-positive hosts, $\mathrm{Cm}^{\mathrm{R}} \mathrm{Gm}^{\mathrm{R}}$ & & Nallapareddy et al. (2006a) \\
\hline pEF13 & pTEX5500ts:pilA-Up, pTEX5500ts with a cloned pilA-Up gene fragment, $\mathrm{Cm}^{\mathrm{R}} \mathrm{Gm}^{\mathrm{R}}$ & & This study \\
\hline pEF15 & $\begin{array}{l}\text { pTEX5500ts:pilA-Up-pilA-Dn, pTEX5500ts with cloned pilA gene fragments flanking } \\
\text { the cat gene, for generating a pilA insertion-deletion mutation, } \mathrm{Cm}^{\mathrm{R}} \mathrm{Gm}^{\mathrm{R}}\end{array}$ & & This study \\
\hline
\end{tabular}


Table 2. Oligonucleotides used in this study

fw, Forward primer; rv, reverse primer.

\begin{tabular}{|ll|}
\hline Primer name & \multicolumn{1}{c|}{ Oligonucleotide sequence $\left(\mathbf{5}^{\prime} \mathbf{- 3} \mathbf{}^{\prime}\right)$} \\
\hline PilA-Up_fw & TTCCGCGGCCGCTATGGCCGACGTCGTC- \\
& GACGCGTGGTTATA-AGTCATTTGAAGAT \\
PilA-Up_rv & CGCGGATCCGCGATAAAACAGTAACA- \\
& AATGGA \\
PilA-Dn_fw & AACTGCAGAACCAATGCATTGGAAATT- \\
& TCGTTAATTCAAAAC \\
PilA-Dn_rv & GCCTTAAGGCCGTTAGGAAATATTACGGAT \\
PilA_fw1 & AACGAAATCCACGCTATTTG \\
PilA_fw2 & TTTACGTATGAAGCTTTTAA \\
PilA_rv1 & ATCAAGGAACGCAATTGAAT \\
PilA_rv2 & GCACAGCTCAAAGCAAACAA \\
Srt1903_fw & CATCAAAAAGATTATCTTTA \\
Srt1903_rv & TAAGAAAAAGCGAATCATGG \\
Srt1905_fw & TAACTAAGTCCATCACCTCC \\
Srt1905_rv & GGTGTTTAAGGAAAATATAG \\
Tuf_fw & TACACGCCACTACGCTCACG \\
Tuf_rv & AGCTCCGTCCATTTGAGCAG \\
Cam_F & TTTAATAAAATTGATTTAGA \\
Cam_R & CCTGAATAGAGTTCATAAAC \\
\end{tabular}

antipeptide polyclonal antibody protocol, by immunizing two specificpathogen-free rabbits with unique 15 aa keyhole limpet haemocyaninconjugated peptides equivalent to the $\mathrm{N}$-terminal regions of PilA (GenBank accession no. ZP_00603529; $\mathrm{H}_{2} \mathrm{~N}$-EDVTQKTPPEKVNITC$\mathrm{CONH}_{2}$ ), PilF (GenBank accession no. ZP_00603526; $\mathrm{H}_{2} \mathrm{~N}-\mathrm{CEKV}-$ IVDPSNPIQNVK-CONH${ }_{2}$ ) and PilE (GenBank accession no. ZP_ 00603527; $\mathrm{H}_{2} \mathrm{~N}-S S D E T V D S S T V D S S E E-\mathrm{CONH}_{2}$ ) of E. faecium strain TX0016 (also termed TX16 or DO) genome sequence. Rabbits were killed on day 87 ; serum was collected and stored at $-25{ }^{\circ} \mathrm{C}$.

Western immunoblot analysis of surface protein extracts. To detect PilA, PilE and PilF pilin protein production, E1165 and E1039 bacteria were grown at 21 and $37^{\circ} \mathrm{C}$ on TSA agar, then resuspended in PBS and harvested by centrifugation $(1560 \mathrm{~g}, 5 \mathrm{~min})$. To extract the surface proteome including pili, cell pellets were suspended in $70 \%$ formic acid and incubated at $65{ }^{\circ} \mathrm{C}$ for $30 \mathrm{~min}$, followed by trichloroacetic acid precipitation and acetone washing (Hendrickx et al., 2008; Ton-That \& Schneewind, 2003). Protein pellets were resuspended in a solution containing $50 \mu \mathrm{l}$ PBS and $50 \mu \mathrm{l}$ sample buffer (100 mM Tris/HCl, $5 \%$ DTT, $2 \%$ SDS, $0.004 \%$ Bromophenol Blue and $20 \%$ glycerol, v/v), and boiled for $5 \mathrm{~min}$. Equal amounts of protein samples of E. faecium E1039 and E1165 were analysed by Western blotting, as described previously (Hendrickx et al., 2008).

Fluorescence-activated cell sorting (FACS) analysis. Bacteria were collected from TSA agar plates and resuspended in RPMI 1640 (Cambrax Bioscience) containing $0.05 \%$ human serum albumin (HSA), to an $\mathrm{OD}_{660}$ of 1.0 (approximately $1 \times 10^{9}$ bacteria $\mathrm{ml}^{-1}$ ). For each sample, $100 \mu \mathrm{l}$ of the cell suspension was pelleted by centrifugation $(6500 \mathrm{~g}, 1 \mathrm{~min})$, and $50 \mu \mathrm{l}$ RPMI/HSA containing 1:100 rabbit anti-PilA immune serum was added. Bacteria were resuspended and incubated on ice. After $30 \mathrm{~min}, 1 \mathrm{ml}$ cold RPMI/ HSA was added, and the bacteria were isolated by centrifugation $(6500 \mathrm{~g}, 1 \mathrm{~min})$. Bacteria were resuspended in $50 \mu \mathrm{l}$ RPMI/HSA containing 1:50 goat anti-rabbit FITC (Sigma-Aldrich), and incubated for $30 \mathrm{~min}$ on ice. Bacteria were washed with $1 \mathrm{ml}$ cold RPMI/ HSA, and after centrifugation $(6500 \mathrm{~g}, 1 \mathrm{~min})$, bacteria were resuspended in $50 \mu \mathrm{RPMI} / \mathrm{HSA}$. Before analyses in the FACSCalibur
(BD Biosciences), bacteria were resuspended in $300 \mu \mathrm{l}$ RPMI/HSA. All measurements were performed in one machine, using the same parameters each time. The data were normalized for bacterial size, and experiments were performed twice. The mean fluorescence was used as a measure of cell-surface-associated PilA. Rabbit pre-immune serum and bacteria incubated without anti-PilA rabbit immune serum were used as negative controls.

Electron microscopy and immunogold labelling. Transmission immunoelectron microscopy (TEM) was performed as described previously, with some modifications (Hendrickx et al., 2007, 2008). In brief, copper grids (mesh Formvar-carbon coated) were incubated for 30 min with the carbon side on a drop of $1 \times 10^{9}$ c.f.u. $\mathrm{ml}^{-1} E$. faecium E1039, E1162 or E1165 cells, to detect surface-exposed PilA, PilE or PilF. Grids were washed three times for $5 \mathrm{~min}$ on drops of $0.02 \mathrm{M}$ glycine in PBS, and subsequently blocked for $30 \mathrm{~min}$ on drops of $1 \%$ BSA in PBS (PBSb). The PilA, PilE and PilF proteins were labelled for $1 \mathrm{~h}$ on drops of undiluted or $1: 100$ diluted specific peptide rabbit immune sera or pre-immune sera in PBSb. Grids were washed four times for $2 \mathrm{~min}$ on drops of $0.1 \%$ BSA in PBS. Surface protein-antibody complexes were labelled by incubation for $20 \mathrm{~min}$ on drops of $1: 70$ diluted protein-A-gold-label $(10 \mathrm{~nm})$ in PBSb. Grids were washed four times for 2 min on drops of PBS, fixed by incubation on drops of $1 \%$ glutaraldehyde in PBS, and washed eight times for 2 min on drops of $\mathrm{H}_{2} \mathrm{O}$. For double-labelling experiments, the labelling was repeated similarly with a $1: 100$ dilution of a second immune serum, and 1:70 diluted protein-A-gold-label (5 nm). After fixation with $1 \%$ glutaraldehyde in PBS, bacteria were stained by incubation of the grids for $5 \mathrm{~min}$ on drops containing $1.8 \%$ methylcellulose (25 mPa s; Sigma-Aldrich) and $0.4 \%$ uranyl acetate (pH4), and they were subsequently air-dried for $10 \mathrm{~min}$. Grids were examined using a JEOL 1010 transmission electron microscope (JEOL Europe).

Mutagenesis of pilA by insertional inactivation. The pilA gene of E. faecium E1165 was disrupted by construction of an insertiondeletion mutation, as described previously (Heikens et al., 2007; Nallapareddy et al., 2006a). In brief, a 397 bp pilA fragment, designated PilA-Up, was amplified from genomic E. faecium E1165 DNA by using primers PilA-Up_fw and PilA-Up_rv, which contain SalI and BamHI restriction sites, respectively (Table 2). The PCR product was digested with SalI and BamHI, and ligated into similarly digested pTEX5500ts, resulting in pEF13. Similarly, a 392 bp fragment, designated PilA-Dn, was amplified by using primers PilADn_fw and PilA-Dn_rv, including restriction sites NsiI and EcoRI incorporated at the $5^{\prime}$ ends of the primers to facilitate directional cloning into pEF13, resulting in pEF15 (Table 1). The pEF15 plasmid was introduced into competent E. faecium E1165 by electroporation. After transformation, gentamicin-resistant colonies were picked and grown overnight at $42{ }^{\circ} \mathrm{C}$ in $\mathrm{BHI}$ broth supplemented with gentamicin; they were subsequently plated on BHI agar plates containing chloramphenicol $\left.(15 \mu \mathrm{g} \mathrm{ml})^{-1}\right)$ and grown at $42{ }^{\circ} \mathrm{C}$. Single-crossover integration into PilA-Up complementary sequences of E. faecium E1165 was confirmed by PCR. Single-crossover mutants were grown overnight for eight serial passages in BHI culture supplemented with consecutive increasing concentrations of chloramphenicol (from 15 to $34 \mu \mathrm{g} \mathrm{ml}^{-1}$ ), at $42{ }^{\circ} \mathrm{C}$, to cure pEF15. Selection for double-crossover recombination was performed by replica plating on BHI agar plates containing either chloramphenicol or gentamicin. The insertional inactivation of the pilA gene in chloramphenicol-resistant gentamicin-susceptible double-crossover mutants was analysed by PCR with primers PilA_fw1 and PilA_rv1, sequencing and Southern hybridization.

Southern blot analysis. Southern blot analysis was performed, as described previously (Hendrickx et al., 2007), on EcoRI-digested chromosomal DNA isolated from E. faecium E1165 and E1165 $\Delta$ pilA 
to confirm disruption of the chromosomal pilA gene. Probes were generated by PCR (in $50 \mu$ volumes) by using primer pairs Cam_F and Cam_R, and PilA_fw2 and PilA_rv2, on the templates pTEX5500ts and chromosomal DNA of E1165, respectively, as shown in Table 2.

Generation of cDNA from total mRNA and PCR. E. faecium isolates E1039 and E1165 were assayed for mRNA expression of the putative srt1903 and srt1905 genes. Total E. faecium RNA isolation, RNA purification (including enzymic digestion of DNA) and cDNA generation were performed as described previously (Hendrickx et al., 2007). cDNA was used as a template for PCR using primer pairs Srt1903_fw and Srt1903_rv, and Srt1905_fw and Srt1905_rv, as listed in Table 2. As an internal control, the housekeeping gene tuf (encoding the elongation factor $\mathrm{Tu}$ ) was amplified by using primers Tuf_fw and Tuf_rv (Table 2). RNA samples not treated with reverse transcriptase were used as controls to detect DNA contamination in the total RNA preparations.

\section{RESULTS}

\section{Characteristics of the proteins putatively encoded from PGC-1 of E. faecium E1165}

The previously identified PGC-1 of E. faecium E1165 (GenBank accession no. EU909697) contains six putative ORFs: orf1901 (pilF), orf1916, orf1902 (pilE), orf1903 (class C sortase), orf1904 (pilA) and orf1905 (class A sortase) (Fig. 1). As previously reported, the orf1901, orf1902 and orf1904 gene products, designated PilF, PilE and PilA, respectively, are predicted to encode cell-wall-associated proteins with LPXTG-like sortase substrate motifs (Hendrickx et al., 2008). The PilE pilin protein is predicted to be a secreted protein (Sillanpaa et al., 2008). Indeed, the SignalP 3.0 server predicted that this protein contains an $\mathrm{N}$-terminal signal sequence. However, visual examination of the amino acid sequence identified six SS repeats and four SD repeats distributed along the protein, and a deviant IVSTG cell-wall-anchor motif, followed by a hydrophobic transmembrane region and a positively charged tail, suggesting that PilE is a putative LPXTG-like surface protein. The IVSTG motif of PilE has been compared with the consensus (FILMPSVY)(AP)X(ATLS)(GAKNS) and NPX(ST)(DGNS) patterns representing distinct sortase substrates (Boekhorst et al., 2005; Janulczyk \& Rasmussen, 2001). However, to date, these putative sortase substrate sequences do not allow the valine (V) on the second position of the IVSTG motif. Furthermore, PilE contains a putative YPKSE pilin motif. Visual examination has revealed that PilA, PilE and PilF are predicted to contain five, two and four $\mathrm{N}$-glycosylation sites $(\mathrm{N}[\mathrm{ST}](\mathrm{P}))$, respectively, suggesting that these pilin subunits may be glycosylated (Power \& Jennings, 2003).

orf1916 putatively encodes a small $6.8 \mathrm{kDa}$ protein containing an N-terminal signal peptide, without a cell wall anchor, and it is possibly a secreted hydrophobic polypeptide.

\section{PilA is a cell-wall-anchored protein in various $E$. faecium isolates}

Recently, we showed that PilA-type pili were assembled on the surface of a clinical E. faecium isolate E1165 (Hendrickx
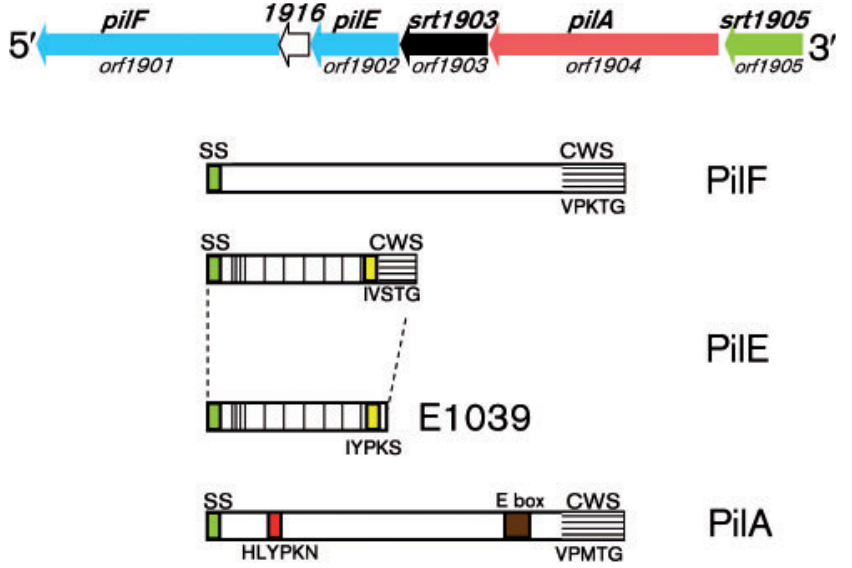

Fig. 1. Schematic representation of the genomic organization of PGC-1 of E. faecium E1165, and E1039 and E1165 gene products (GenBank accession nos NZ_ACO501000003 and EU909697). The positions and presumed directions of transcription of ORFs are indicated by arrows. The ORF numbers are given below the arrows, while the gene names are given in bold above the arrows. The blue arrows indicate minor pilin proteins, and the white arrow indicates a gene with unknown function. The red arrow indicates the putative major pilin subunit. The black and green arrows indicate class $C$ and class A sortase genes, respectively. The putative LPXTG-like surface proteins are shown below PGC-1. SS, signal sequence. The pilin and pilin-like motifs are shown in red and yellow, respectively. The repeats in PilE are shown by vertical black lines.

et al., 2008). To analyse the display of PilA on the surfaces of other E. faecium isolates, three clinical (E1162, E1360 and E1391) and four non-clinical (E128, E1039, E1133 and E1674) isolates were selected, and PCR and DNA-DNA hybridizations confirmed the presence of pilA in these strains, except in E128, which was used as a negative control. Based on MLST, the E1162, E1165, E1360 and E1391 isolates belong to the polyclonal hospital-acquired genetic lineage, whereas E128, E1039, E1133 and E1674 are of non-hospital-acquired origin (Table 1).

Surface exposure of PilA was demonstrated by FACS analysis in five pilA-positive strains grown on TSA agar at $37^{\circ} \mathrm{C}$. PilA protein levels varied among the isolates analysed, with the highest level found at the surface of E1039 (Fig. 2). Strain E1360 displayed a low mean fluorescence, which was comparable to that of the pilA-deficient strain (E128), suggesting that PilA was not produced in this isolate. The flow cytometry analyses were confirmed by TEM on negatively stained and anti-PilA immunogold-labelled bacteria. Gold particles were associated with the bacterial cell wall at different levels, but were not associated with pilus-like structures, suggesting differential PilA pilus assembly among E. faecium isolates (data not shown). Surface exposure of PilA at the cell wall of E1039 was the highest compared with the other isolates, and was therefore subjected to further analyses in this study. 


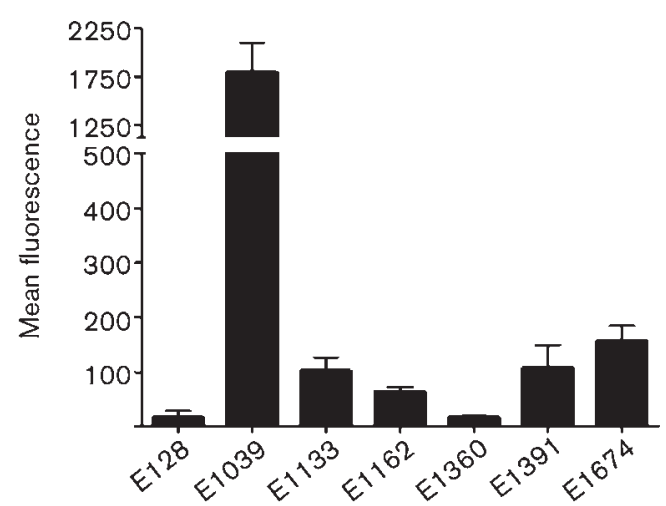

Fig. 2. Flow cytometry analysis of surface display of PilA on $E$. faecium strains. Three clinical (E1162, E1360, and E1391) and four non-clinical (E128, E1039, E1133 and E1674) isolates were grown on TSA at $37{ }^{\circ} \mathrm{C}$ in aerobic conditions to analyse PilA protein levels by flow cytometry. A representative of two independent experiments is shown. Bars, SD.

\section{Comparison of the PGC-1 sequence of E1039 and E1165 reveals heterogeneity in orf1916 and pilE}

The sequence of PGC-1 of E. faecium strain E1039 (GenBank accession no. NZ_ACOS01000003) (van Schaik et al., 2010), which did not assemble PilA pili, was compared with the recently sequenced PGC-1 of E. faecium E1165, which is a strain that produces PilA pili. In general, the proteins putatively encoded from the ORFs from PGC-1 of E1039 and E1165 exhibited a very high overall similarity of $>97 \%$ (Table 3). The organization and transcriptional direction of the ORFs in this PGC were similar to each other, demonstrating that PGC-1 is conserved among $E$. faecium E1039 and E1165. The sortase genes srt1903 and srt1905 of E1039 and E1165 did not contain mutations leading to amino acid changes in the active site amino acid residues or the TLxTC catalytic pocket (Ilangovan et al., 2001; Mazmanian et al., 1999). Furthermore, conserved elements required for pilus assembly, such as the CWS of the putative minor pilin protein PilF, the HLYPKN pilin

Table 3. Similarities between the proteins of PGC-1 of $E$. faecium E1039 and E1165

\begin{tabular}{|llc|}
\hline $\begin{array}{l}\text { PGC-1 } \\
\text { protein }\end{array}$ & Putative function & $\begin{array}{c}\text { Amino acid } \\
\text { identity (\%) }\end{array}$ \\
\hline Srt1905 & $\begin{array}{c}\text { Class A housekeeping } \\
\text { sortase }\end{array}$ & 98.7 \\
PilA & Major pilin subunit \\
Srt1903 & Class C pilus-dedicated & 98.9 \\
& sortase & 98.8 \\
PilE & PilF linker protein & 97.8 \\
Orf1916 & Secreted peptide & 97.7 \\
PilF & Minor pilin subunit & 99.3 \\
\hline
\end{tabular}

motif, the E box sequence and the CWS of the major PilA pilin subunit, were identical among the E1039 and E1165 strains. The largest sequence heterogeneity in PGC-1 was found in the putative PilE and Orf1916 proteins. PilE of E1039 did not contain a CWS, but it did contain a Cterminal IYPKS sequence, which resembles a pilin motif (Fig. 1). Orf1916 contained two amino acid substitutions in the hydrophobic polypeptide.

\section{Display of PilA, PilE and PilF pilin proteins on the surface of $E$. faecium E1039 under different environmental conditions}

To show the presence of the PilA, PilE and PilF proteins at the surface of E. faecium E1039, TEM was performed on negatively stained and immunogold-labelled E1039 bacteria grown on TSA agar at 21 and $37{ }^{\circ} \mathrm{C}$. Using specific peptide antisera directed against PilA, PilE and PilF, gold particles were associated with the cell wall of strain E1039 at $21{ }^{\circ} \mathrm{C}$ (Fig. 3a-c) and at lower levels at $37{ }^{\circ} \mathrm{C}$ (Fig. 3b, d, f). The PilA, PilE and PilF proteins were equally distributed in high densities at the surface of the cells. Although the PilE protein in E1039 lacks the deviant IVSTG putative sortase substrate motif, the protein was present at the cell wall of E1039. No gold particles were associated with the cell wall when cells were incubated with the individual rabbit preimmune sera (Fig. 3d) or when immune sera were omitted (conjugate control; protein-A-gold) (Fig. 3e).

Since the DNA sequences of PGC-1 of E1039 and E1165 were highly similar, we hypothesized that particular environmental conditions relevant for the in vivo lifestyle of E. faecium may induce PilA pilus assembly in the nonhospital-acquired E1039 isolate. Therefore, E1039 and E1165 strains were grown under anaerobic conditions on TSA and BHI agar at $37^{\circ} \mathrm{C}$, in aerobic conditions on BHI at $37^{\circ} \mathrm{C}$, and on TSA and BHI agar at the elevated temperature of $42{ }^{\circ} \mathrm{C}$, and in filter-sterilized human male urine at $37^{\circ} \mathrm{C}$. The E1039 and E1165 cells grown under these different conditions were harvested, and subjected to TEM analysis using anti-PilA immune serum. Strain E1165 assembled PilA-type pili at the surface during all the different growth conditions; however, in strain E1039, antiPilA immune serum labelled PilA at the surface, but PilA pilus polymerization was not induced (data not shown).

\section{PilF, but not PilE, is associated with pili in E. faecium E1165}

To corroborate whether the PilE and PilF pilin proteins were associated with the cell wall and pili, respectively, single-labelling TEM experiments were performed on $E$. faecium E1165 cells grown on TSA agar at 21 and $37^{\circ} \mathrm{C}$. In the TEM experiments, cells were labelled with either rabbit anti-PilE or anti-PilF immune serum, followed by proteinA-gold $(10 \mathrm{~nm})$. In contrast with E1039, no gold particles bound to the cell wall or to the pili of E1165 when cells were grown at either temperature and incubated with 

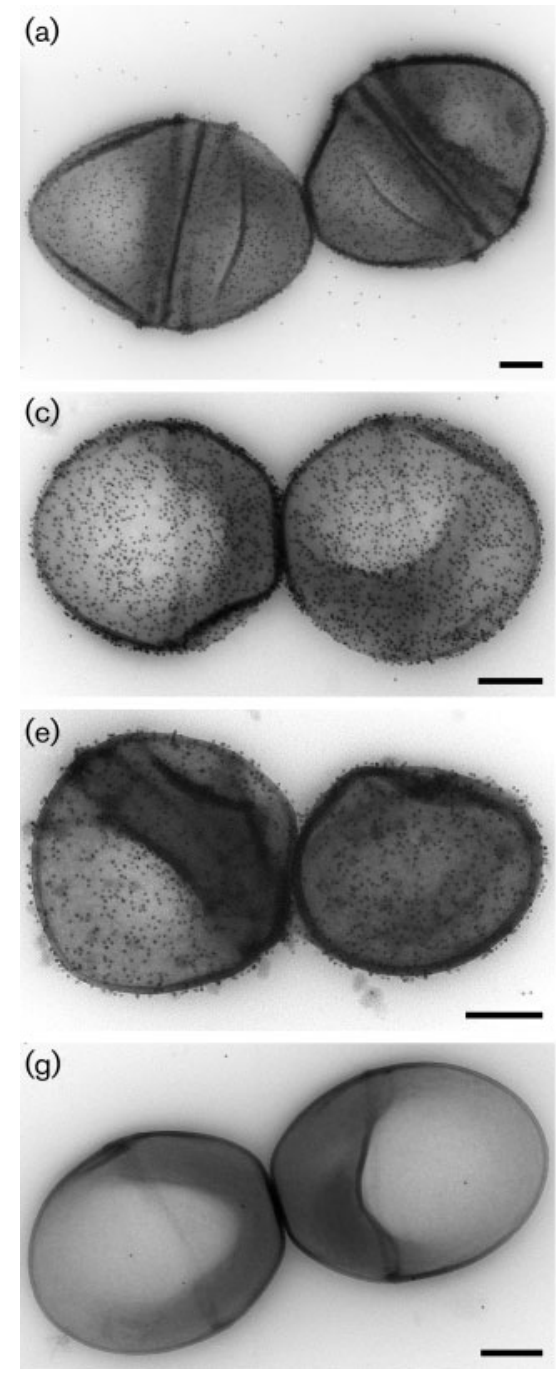
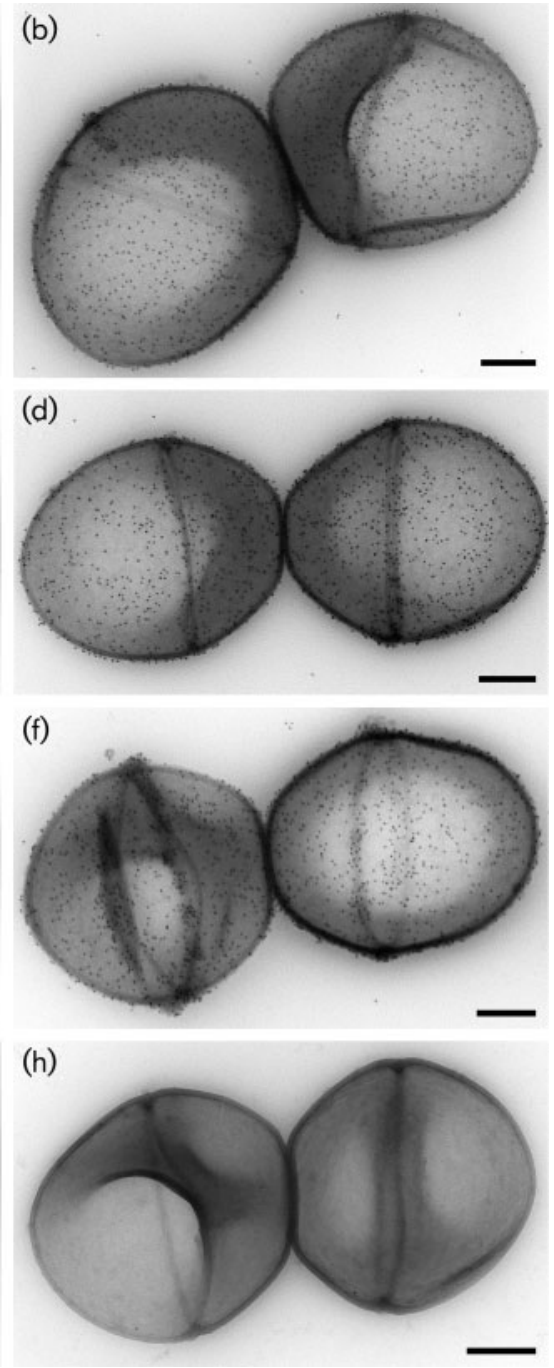

Fig. 3. TEM micrographs of E. faecium E1039. Strain E1039 was grown at 21 or $37^{\circ} \mathrm{C}$ and labelled individually with rabbit anti-PilA, -PilE or -PilF immune serum, followed by protein-Agold $(10 \mathrm{~nm})$. Samples were negatively stained. Temperatures and serum labels were as follows: (a) $21^{\circ} \mathrm{C}$, anti-PilA serum; (b) $37{ }^{\circ} \mathrm{C}$, anti-PilA serum; (c) $21^{\circ} \mathrm{C}$, anti-PilE serum; (d) $37{ }^{\circ} \mathrm{C}$, anti-PilE serum; (e) $21^{\circ} \mathrm{C}$, anti-PilF serum; (f) $37{ }^{\circ} \mathrm{C}$, anti-PilF serum. (g) Representative micrograph of E1039 incubated with rabbit pre-immune serum followed by protein-A-gold $(10 \mathrm{~nm})$. (h) E1039 incubated with protein-A-gold only (conjugate control). Bars, $200 \mathrm{~nm}$.
anti-PilE serum (data not shown). Using anti-PilF serum, PilF was associated with pili present at the poles in $40 \%$ of the cells (Fig. 4a), and cells without pili contained cell-wallassociated PilF or did not express PilF at all. Gold particles were equally distributed along pilus shafts at $37{ }^{\circ} \mathrm{C}$ (Fig. 4b), and were associated with pilus tips (Fig. 4c) and the base of the pilus (Fig. 4d). PilF was associated with the cell wall at $21{ }^{\circ} \mathrm{C}$ (Fig. 4e) and did not assemble into pili. Rabbit pre-immune serum did not react with piluslike structures or with the cell wall (Fig. 4f).

\section{PilF is assembled into PilA-type pili of E. faecium}

To investigate whether the PilF pilin subunit was associated with PilA-type pili, a double-labelling TEM experiment was performed. E. faecium E1165 cells were grown at $37{ }^{\circ} \mathrm{C}$, and initially labelled with rabbit anti-PilA immune serum, followed by protein-A-gold ( $5 \mathrm{~nm}$ ). They were then fixed, and subsequently incubated with anti-PilF serum, followed by protein-A-gold $(10 \mathrm{~nm})$. TEM analysis confirmed that both PilA and PilF immune sera bound to the same type of pili (Fig. 5a). We showed that in PilA-type pili, PilA forms the major pilin subunit and PilF forms a minor subunit, which is also positioned at the pilus tip. Multiple (typically 3-5 gold particles) PilF pilin subunits were also positioned at the base of some PilA pili (Fig. 5b). PilF was also found to be distributed along the PilA pilus shaft.

To confirm that PilF is assembled into PilA-type pili, the pilA gene was inactivated by an insertion-deletion mutation in the multiresistant clinical E. faecium E1165 isolate. Correct insertion-deletion mutation in the pilA gene was confirmed by PCR, sequencing and Southern hybridization, and it was demonstrated that in the doublecrossover E1165 $\Delta$ pilA mutant, a part of the pilA gene was replaced by the chloramphenicol (cat) cassette (data not shown). Subsequent TEM analysis demonstrated that in the E1165 $\Delta$ pilA strain, PilF bound to the cell surface only, and was not associated with pili (Fig. 5c). In addition, Western blotting performed on surface protein extracts of wild-type and E1165 $\Delta$ pilA cells revealed the absence of high-molecular-mass PilF pilin species (data not shown). 

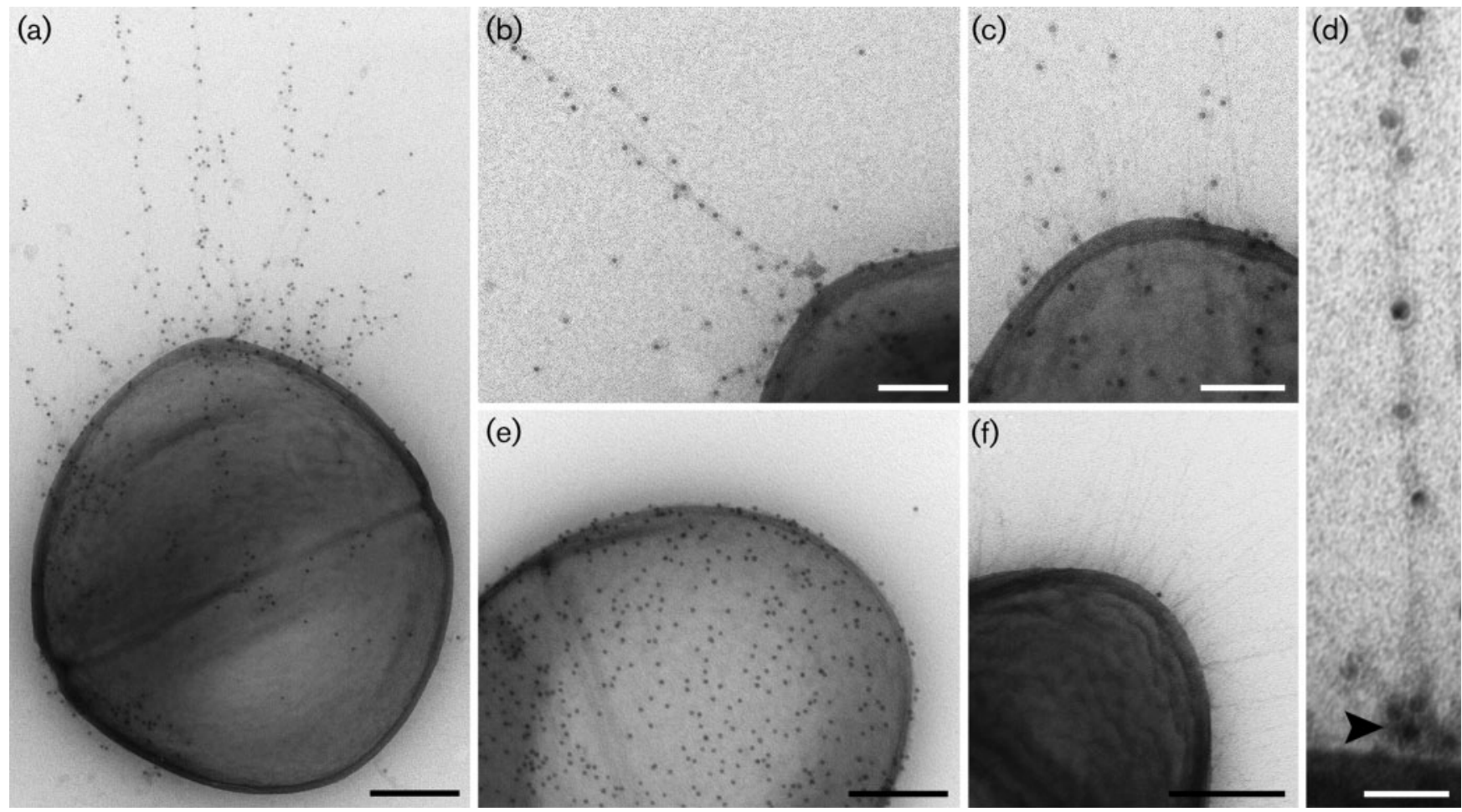

(f)
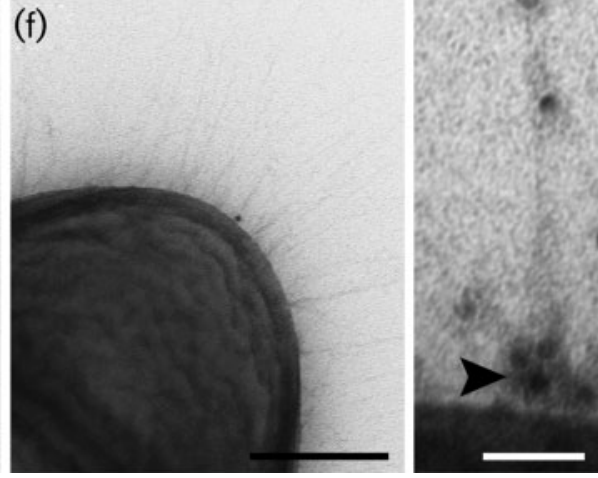

Fig. 4. TEM micrographs of PilF associated with pili of $E$. faecium $\mathrm{E} 1165$. E1165 cells were grown at 21 or $37{ }^{\circ} \mathrm{C}$, and labelled individually with rabbit anti-PilF immune serum, followed by protein-A-gold $(10 \mathrm{~nm}$ ); samples were negatively stained. (a) PilF is associated with pili at the pole of the cell (bar, $200 \mathrm{~nm}$ ). (b, c) Detail of E1165 grown at $37{ }^{\circ} \mathrm{C}$, showing that PilF is associated along a thin pilus of $1 \mu \mathrm{m}$ (b) or that PilF is positioned at the tip of pilus-like structures (c) (bars, $100 \mathrm{~nm}$ ). (d) Detail of a pilus, showing that PilF is positioned at the tip, along the pilus shaft, and present in an elevated concentration at the base of the pilus (arrow) (bar, $40 \mathrm{~nm}$ ). (e) E1165 grown at $21^{\circ} \mathrm{C}$, showing that PilF is associated at the cell wall (bar, $200 \mathrm{~nm}$ ). (f) E1165 incubated with rabbit pre-immune serum, followed by protein-A-gold $(10 \mathrm{~nm})($ bar, $200 \mathrm{~nm})$.

\section{Differential surface display of PilA, PilE and PilF in E. faecium E1039 and E1165}

To analyse the differences in deposition of the PilA, PilE and PilF LPXTG-like proteins at the surfaces of E. faecium strains E1039 and E1165 in more detail, Western blotting was performed on surface protein extracts of cells grown on TSA plates at 21 and $37^{\circ} \mathrm{C}$. This confirmed our previous results showing that PilA assembled into high-molecular-mass pilus-like structures in E1165 at $37^{\circ} \mathrm{C}$, whereas the monomeric subunit was produced at the predicted molecular mass of $64.8 \mathrm{kDa}$ at the surface when cells were grown at $21{ }^{\circ} \mathrm{C}$ (Fig. 6a). In contrast, PilA did not assemble into highmolecular-mass pili in E. faecium E1039, but displayed at the surface at $37{ }^{\circ} \mathrm{C}$; protein levels were higher at $21{ }^{\circ} \mathrm{C}$.

Anti-PilE serum reacted with a $\sim 90 \mathrm{kDa}$ protein band in surface protein extracts of E1039 at $21{ }^{\circ} \mathrm{C}$, and at lower levels at $37{ }^{\circ} \mathrm{C}$, while the PilE protein was not detected in cell wall extracts of E1165 at either temperature (Fig. 6b). The observed molecular mass of PilE did not correlate with the predicted molecular mass of $22.8 \mathrm{kDa}$ (after posttranslational modification), suggesting association of PilE with another protein. In strain E1039, the anti-PilF sera reacted with three bands of $75, \sim 90$ and $\sim 100 \mathrm{kDa}$ at $37{ }^{\circ} \mathrm{C}$, and with one predominant protein band of $\sim 75 \mathrm{kDa}$ at $21{ }^{\circ} \mathrm{C}$. Anti-PilF serum reacted with a smear of proteinreactive material in E1165, which ranged into the stacking gel, suggesting that the PilF pilin protein is associated with pili (Fig. 6c). The monomeric PilF subunit was produced at predicted molecular mass of $70.4 \mathrm{kDa}$ (after post-translational modification) at $21{ }^{\circ} \mathrm{C}$. Pre-immune sera were used as negative controls, and did not react with any of the protein products.

\section{mRNA expression of the housekeeping sortase and pilus-dedicated sortase of PGC-1}

Total RNA was isolated from the clinical E1165 and community-derived E1039 E. faecium strains after growth on TSA plates at 21 and $37{ }^{\circ} \mathrm{C}$. mRNA expression of srt1903 (pilus-dedicated sortase), srt1905 (housekeeping sortase) and tuf (internal housekeeping control) genes was analysed by PCR on cDNA generated from total mRNA. mRNA transcripts of srt1903 and srt1905 were detected in comparable levels in the E1039 and E1165 strains at both temperatures (Fig. 7), indicating that strain- or 


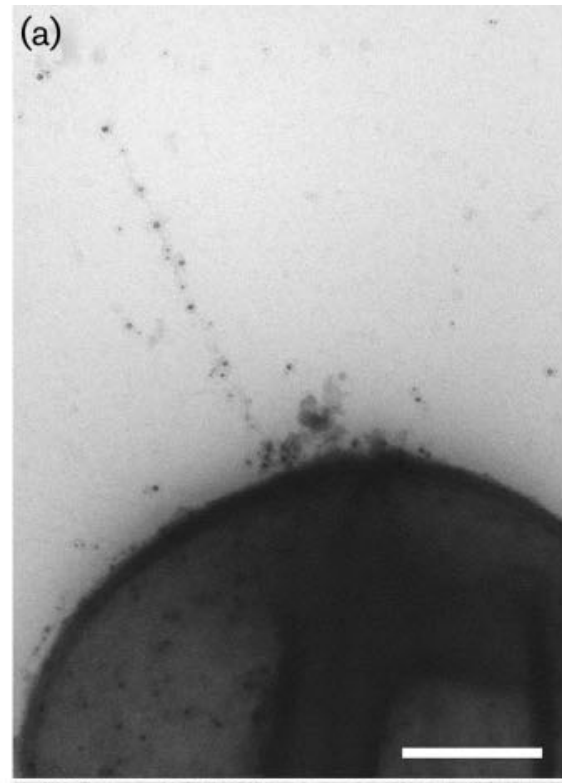

(b)

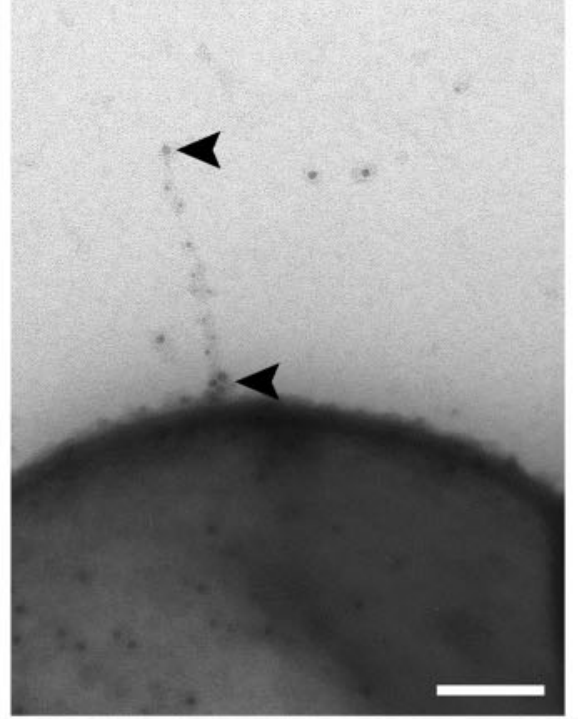

(c)

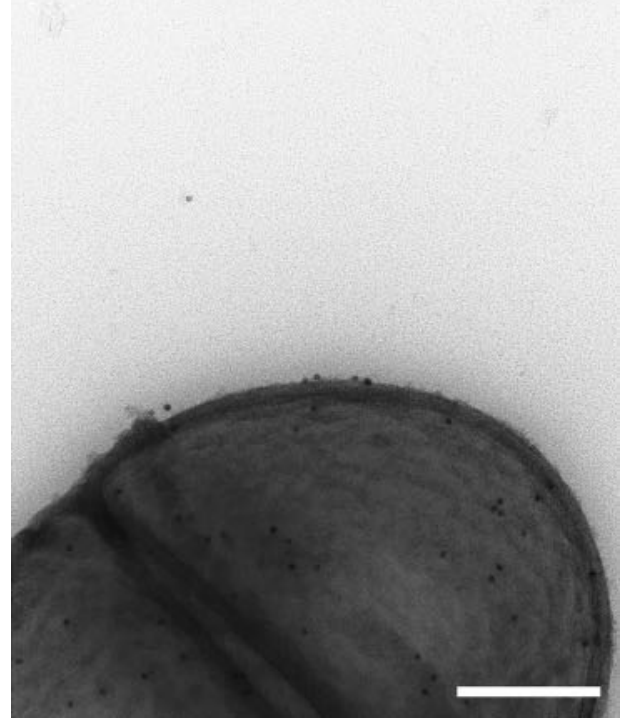

temperature-dependent differences in expression of the putative-dedicated sortases do not cause the observed differences in pilus assembly between E1039 and E1165. The control tuf housekeeping gene was expressed in all isolates analysed. No DNA contamination was detected in control tuf reactions of the DNase-treated total mRNA preparations in which the reverse transcriptase reaction was omitted.

\section{DISCUSSION}

E. faecium isolates of clinical and non-clinical origin express PilA-type pili differently, showing the complexity of pilus assembly and pilin protein production among $E$. faecium isolates from different sources, and of distinct genetic background. More specifically, we demonstrated the differential assembly of PilA-type pili at the surface of a human E. faecium clinical hospital-acquired bloodstream isolate (E1165) and a community-derived stool isolate (E1039) grown at 21 and $37^{\circ} \mathrm{C}$. While PilA and PilF proteins were associated with the cell wall of E1165 grown at $21{ }^{\circ} \mathrm{C}$, and assembled into high-molecular-mass pilin species at $37^{\circ} \mathrm{C}$, PilA, PilE and PilF pilin proteins appeared to be cell-wall-associated and not pilus-associated in E1039, when grown at 37 or $21{ }^{\circ} \mathrm{C}$. At $21{ }^{\circ} \mathrm{C}$, all three proteins were displayed at the surface in higher levels. Hence, in the community-derived stool isolate, not only was pilus assembly abrogated, but also the level of surface protein production was differentially regulated when compared with strain E1165. mRNA expression analysis of the pilus-dedicated sortase and housekeeping sortase genes located in PGC-1 revealed comparable levels of transcripts, suggesting that the differential PilA pilus assembly is controlled post-transcriptionally. Which environmental conditions that are relevant for the lifestyle of $E$. faecium induce PilA pilus polymerization in the E1039 strain or in the other tested E. faecium isolates, such as growth in the presence of microbial communities in the gastrointestinal tract, in bile, serum or blood, or growth under fluctuating $\mathrm{pH}$ or carbon dioxide concentrations, remain to be elucidated.

Double-labelling TEM analysis of the wild-type E1165 and the isogenic pilA mutant demonstrated that the PilA protein is the major component of PilA-type pili, and that it forms the pilus backbone. TEM analyses also showed that the PilF protein was found at the pilus base, was equally distributed along the pilus shaft and was associated with the PilA pilus tip in the clinical E. faecium E1165 strain at

Fig. 5. TEM micrographs of PilF associated with PilA-type pili. $E$. faecium E1165 was grown at $37{ }^{\circ} \mathrm{C}$ and labelled with rabbit antiPilA immune serum, followed by protein-A-gold ( $5 \mathrm{~nm}$ particles). Cells were fixed and consecutively labelled using anti-PilF immune serum, followed by protein-A-gold (10 nm particles). (a) Distribution of PilF along the PilA pilus shaft. (b) PilF (arrowheads) is positioned at the PilA pilus tip and at the pilus base. (c) An isogenic pilA mutant, E1165 $\Delta$ pilA did not display PilF/PilA pili. Bars, 200 nm. 


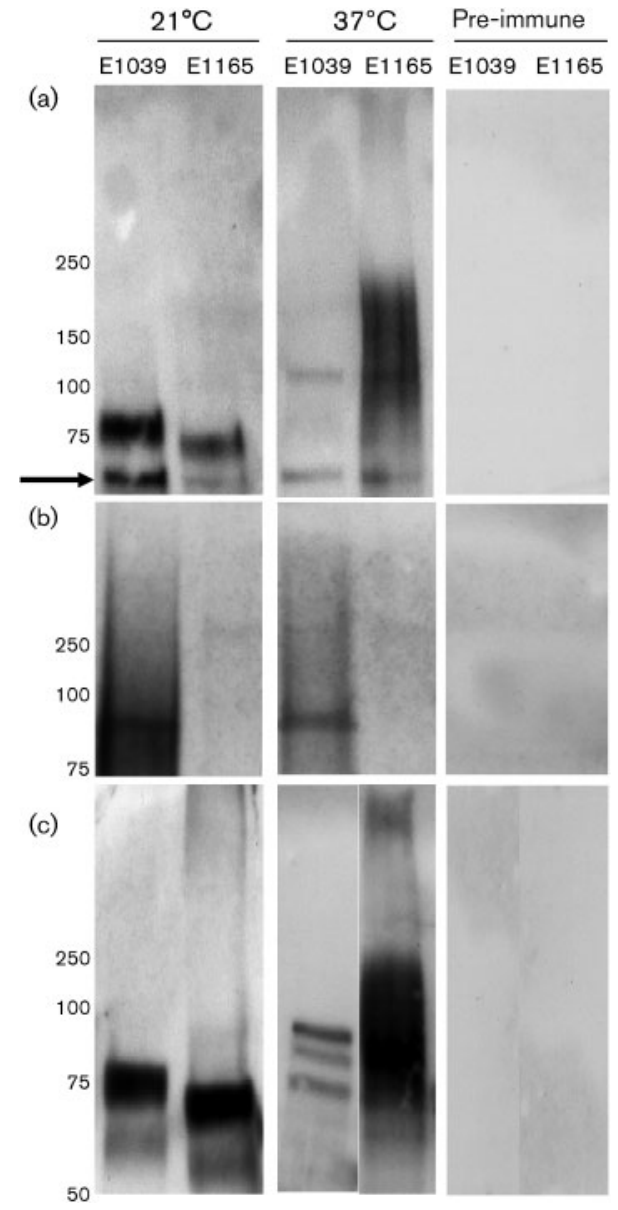

Fig. 6. Western blots of surface protein extracts of $E$. faecium E1039 and E1165 grown on TSA agar at 21 and $37^{\circ} \mathrm{C}$. Blots were probed with rabbit anti-PilA (a), rabbit anti-PilE (b) and rabbit anti-PilF immune sera (c) (left lanes), and with the respective preimmune sera (right lanes), followed by goat anti-rabbit lgGhorseradish-peroxidase conjugate. Molecular mass values ( $\mathrm{BDa}$ ) are shown on the left. Predicted monomeric PilA $(64.8 \mathrm{kDa})$ is indicated by an arrow.
$37{ }^{\circ} \mathrm{C}$. Therefore, PilF was designated a minor pilin subunit, which may represent an adhesin that potentially binds to biotic or abiotic surfaces. It has been demonstrated that the sorting signal IPNTG of the BcpB minor pilin subunit (which lacks a pilin motif) of Bacillus cereus is distinct from the LPXTG sorting signal of the major pilin subunit BcpA, and is specifically cleaved by the pilusdedicated sortase, and not by the housekeeping sortase, thereby initiating polymerization (Budzik et al., 2009). This sortase-specific cleavage of the minor pilin protein indicates that $\mathrm{BcpB}$ is positioned at the $\mathrm{BcpA}$ pilus tip of $B$. cereus. Initiation of PilA pilus polymerization may be more complex in E. faecium. The fact that both PilA and PilF proteins harbour VPXTG sorting motifs suggests that another signal, in addition to the sorting substrate motif that determines sortase specificity, is required to initiate pilus polymerization in E. faecium.

Recently, it has been shown that the minor pilin subunit $(\mathrm{SpaB})$ is required to stop SpaA-type pilus polymerization of Corynebacterium diphtheriae, and to anchor pilus polymers to the cell wall through incorporation of the minor pilin subunit in the base of the growing pilus shaft (Mandlik et al., 2008). Localization of PilF at the base of PilA pili, as observed in our study, suggests that PilF may also be required for termination of pilus polymerization. However, construction of a pilF mutant will be needed to provide evidence for this hypothesis.

The role of PilE in pilus biogenesis remains unresolved and requires further characterization. In E1165, PilE protein was not associated with PilA-type pili or expressed at the cell wall at 21 or $37{ }^{\circ} \mathrm{C}$. PilE, which lacked the deviant IVSTG motif in E1039, is an SE-rich protein, and the SD repeats or S-rich repeat domains of multiple MSCRAMMs are predicted to adopt a long coiled structure to expose the ligand-binding domain to the extracellular environment (Deivanayagam et al., 2000; Hartford et al., 1997). The presence of SD repeats and S-rich repeats in PilE suggests that this protein may adopt a comparable structure. Since cell-surface exposure of PilE correlated with absence of assembled pili in E1039, we suggest that surface deposition of PilE abrogates pilus biogenesis. To prove this, conclusive

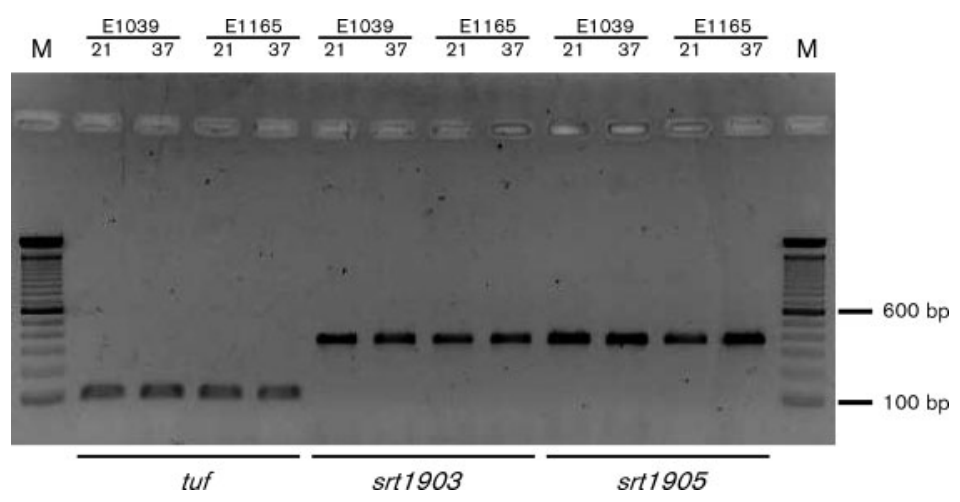

Fig. 7. $m R N A$ expression of sortase genes stt1903 and srt1905 of PGC-1. The mRNAs of the community-derived isolate E1039 and the clinical isolate E1165, grown at 21 and $37{ }^{\circ} \mathrm{C}$, are shown. Control tuf RT-PCRs (internal housekeeping control) with $E$. faecium-specific tuf primers were all positive. mRNA expression levels were similar for the srt1903 pilusdedicated sortase gene (srt1903) and the srt1905 housekeeping sortase gene of the two strains at both temperatures. The results are presented as PCR products separated on an ethidium-bromide-stained $1.0 \%$ agarose gel. M, Molecular mass markers. 
experimental evidence has to be obtained using additional knockout mutations in genes of this PGC. However, to date, attempts to construct isogenic pilE and pilF mutants using the pTEX5500ts vector have failed.

Only in 2005, the first Gram-positive PGCs were identified and the roles of the different proteins encoded by these PGCs were elucidated. Our study has disclosed novel findings with respect to temperature- and strain-dependent regulation, as well as differential assembly of pili at the surface of a clinically relevant Gram-positive species. Increasing the knowledge of pilus assembly will increase our understanding of the different steps implicated in Gram-positive pathogenesis in general and in E. faecium in particular. Understanding colonization and virulence factors is important since the incidence of E. faecium infections with acquired resistance to the three commonly used classes of antimicrobials, i.e. aminoglycosides, $\beta$ lactams and glycopeptides, continues to increase (Werner et al., 2008). Treatment of these infections will become even more challenging when resistance against antibiotics from other, recently introduced, classes of antimicrobials, such as daptomycin and linezolid, emerges (Aksoy \& Unal, 2008; Kainer et al., 2007; Montero et al., 2008). Therefore, novel alternative treatment and prevention strategies such as passive or active immunization are urgently required. Gram-positive pilin proteins may be promising novel targets for immune therapy (Gianfaldoni et al., 2007; Mora et al., 2005).

\section{ACKNOWLEDGEMENTS}

We thank Marc van Peski and René Scriwanek for help with the TEM micrographs. This work was supported by The Netherlands Organization for Health, Research and Development (ZonMW research grant 6100.008), by a grant from the European Union Sixth Framework Programme, under contract LSHE-CT-2007-037410 and an NWO Veni grant (916.86.044). Part of this study was presented as a poster at the 109th General Meeting of the American Society for Microbiology, Philadelphia, PA, 17-21 May 2009 (abstract 09-GM-A-2001-ASM).

\section{REFERENCES}

Abbot, E. L., Smith, W. D., Siou, G. P., Chiriboga, C., Smith, R. J., Wilson, J. A., Hirst, B. H. \& Kehoe, M. A. (2007). Pili mediate specific adhesion of Streptococcus pyogenes to human tonsil and skin. Cell Microbiol 9, 1822-1833.

Aksoy, D. Y. \& Unal, S. (2008). New antimicrobial agents for the treatment of Gram-positive bacterial infections. Clin Microbiol Infect 14, 411-420.

Boekhorst, J., de Been, M. W., Kleerebezem, M. \& Siezen, R. J. (2005). Genome-wide detection and analysis of cell wall-bound proteins with LPxTG-like sorting motifs. J Bacteriol 187, 4928-4934.

Budzik, J. M., Oh, S. Y. \& Schneewind, O. (2009). Sortase D forms the covalent bond that links BcpB to the tip of Bacillus cereus pili. J Biol Chem 284, 12989-12997.

Deivanayagam, C. C., Rich, R. L., Carson, M., Owens, R. T., Danthuluri, S., Bice, T., Hook, M. \& Narayana, S. V. (2000). Novel fold and assembly of the repetitive B region of the Staphylococcus aureus collagen-binding surface protein. Structure 8, 67-78.

Dramsi, S., Caliot, E., Bonne, I., Guadagnini, S., Prevost, M. C., Kojadinovic, M., Lalioui, L., Poyart, C. \& Trieu-Cuot, P. (2006). Assembly and role of pili in group B streptococci. Mol Microbiol 60, 1401-1413.

Gaspar, A. H. \& Ton-That, H. (2006). Assembly of distinct pilus structures on the surface of Corynebacterium diphtheriae. J Bacteriol 188, 1526-1533.

Gianfaldoni, C., Censini, S., Hilleringmann, M., Moschioni, M., Facciotti, C., Pansegrau, W., Masignani, V., Covacci, A., Rappuoli, R. \& other authors (2007). Streptococcus pneumoniae pilus subunits protect mice against lethal challenge. Infect Immun 75, 1059-1062.

Hanahan, D. (1983). Studies on transformation of Escherichia coli with plasmids. J Mol Biol 166, 557-580.

Hartford, O., Francois, P., Vaudaux, P. \& Foster, T. J. (1997). The dipeptide repeat region of the fibrinogen-binding protein (clumping factor) is required for functional expression of the fibrinogen-binding domain on the Staphylococcus aureus cell surface. Mol Microbiol 25, 1065-1076.

Heikens, E., Bonten, M. J. \& Willems, R. J. (2007). Enterococcal surface protein Esp is important for biofilm formation of Enterococcus faecium E1162. J Bacteriol 189, 8233-8240.

Hendrickx, A. P., Van Wamel, W. J., Posthuma, G., Bonten, M. J. \& Willems, R. J. (2007). Five genes encoding surface-exposed LPXTG proteins are enriched in hospital-adapted Enterococcus faecium clonal complex 17 isolates. J Bacteriol 189, 8321-8332.

Hendrickx, A. P., Bonten, M. J., van Luit-Asbroek, M., Schapendonk, C. M., Kragten, A. H. \& Willems, R. J. (2008). Expression of two distinct types of pili by a hospital-acquired Enterococcus faecium isolate. Microbiology 154, 3212-3223.

Hendrickx, A. P., van Luit-Asbroek, M., Schapendonk, C. M., Van Wamel, W. J., Braat, J. C., Wijnands, L. M., Bonten, M. J. \& Willems, R. J. (2009a). SgrA, a nidogen-binding LPXTG surface adhesin implicated in biofilm formation, and EcbA, a collagen binding MSCRAMM, are two novel adhesins of hospital-acquired Enterococcus faecium. Infect Immun 77, 5097-5106.

Hendrickx, A. P., Willems, R. J., Bonten, M. J. \& van Schaik, W. (2009b). LPXTG surface proteins of enterococci. Trends Microbiol 17, 423-430.

Hidron, A. I., Edwards, J. R., Patel, J., Horan, T. C., Sievert, D. M., Pollock, D. A. \& Fridkin, S. K. (2008). NHSN annual update: antimicrobial-resistant pathogens associated with healthcare-associated infections: annual summary of data reported to the National Healthcare Safety Network at the Centers for Disease Control and Prevention, 2006-2007. Infect Control Hosp Epidemiol 29, 996-1011.

Ilangovan, U., Ton-That, H., Iwahara, J., Schneewind, O. \& Clubb, R. T. (2001). Structure of sortase, the transpeptidase that anchors proteins to the cell wall of Staphylococcus aureus. Proc Natl Acad Sci U S A 98 , 6056-6061.

Janulczyk, R. \& Rasmussen, M. (2001). Improved pattern for genome-based screening identifies novel cell wall-attached proteins in Gram-positive bacteria. Infect Immun 69, 4019-4026.

Kainer, M. A., Devasia, R. A., Jones, T. F., Simmons, B. P., Melton, K., Chow, S., Broyles, J., Moore, K. L., Craig, A. S. \& Schaffner, W. (2007). Response to emerging infection leading to outbreak of linezolid-resistant enterococci. Emerg Infect Dis 13, 1024-1030.

Leavis, H., Top, J., Shankar, N., Borgen, K., Bonten, M., van Embden, J. \& Willems, R. J. (2004). A novel putative enterococcal pathogenicity island linked to the esp virulence gene of Enterococcus faecium and associated with epidemicity. J Bacteriol 186, 672-682.

Leavis, H. L., Willems, R. J., Top, J. \& Bonten, M. J. (2006). High-level ciprofloxacin resistance from point mutations in $g y r A$ and parC 
confined to global hospital-adapted clonal lineage CC17 of Enterococcus faecium. J Clin Microbiol 44, 1059-1064.

Leavis, H. L., Willems, R. J., Van Wamel, W. J., Schuren, F. H., Caspers, M. P. \& Bonten, M. J. (2007). Insertion sequence-driven diversification creates a globally dispersed emerging multiresistant subspecies of E. faecium. PLoS Pathog 3, e7.

Mandlik, A., Das, A. \& Ton-That, H. (2008). The molecular switch that activates the cell wall anchoring step of pilus assembly in Grampositive bacteria. Proc Natl Acad Sci U S A 105, 14147-14152.

Marraffini, L. A. \& Schneewind, O. (2006). Targeting proteins to the cell wall of sporulating Bacillus anthracis. Mol Microbiol 62, 14021417.

Mazmanian, S. K., Liu, G., Ton-That, H. \& Schneewind, O. (1999). Staphylococcus aureus sortase, an enzyme that anchors surface proteins to the cell wall. Science 285, 760-763.

Montero, C. I., Stock, F. \& Murray, P. R. (2008). Mechanisms of resistance to daptomycin in Enterococcus faecium. Antimicrob Agents Chemother 52, 1167-1170.

Mora, M., Bensi, G., Capo, S., Falugi, F., Zingaretti, C., Manetti, A. G., Maggi, T., Taddei, A. R., Grandi, G. \& Telford, J. L. (2005). Group A Streptococcus produce pilus-like structures containing protective antigens and Lancefield T antigens. Proc Natl Acad Sci U S A 102, 15641-15646.

Murdoch, D. R., Mirrett, S., Harrell, L. J., Monahan, J. S. \& Reller, L. B. (2002). Sequential emergence of antibiotic resistance in enterococcal bloodstream isolates over 25 years. Antimicrob Agents Chemother 46, 3676-3678.

Murray, B. E. (2000). Vancomycin-resistant enterococcal infections. $N$ Engl J Med 342, 710-721.

Nallapareddy, S. R., Singh, K. V. \& Murray, B. E. (2006a). Construction of improved temperature-sensitive and mobilizable vectors and their use for constructing mutations in the adhesinencoding acm gene of poorly transformable clinical Enterococcus faecium strains. Appl Environ Microbiol 72, 334-345.

Nallapareddy, S. R., Singh, K. V., Sillanpaa, J., Garsin, D. A., Hook, M., Erlandsen, S. L. \& Murray, B. E. (2006b). Endocarditis and biofilm-associated pili of Enterococcus faecalis. J Clin Invest 116, 2799-2807.

Nelson, A. L., Ries, J., Bagnoli, F., Dahlberg, S., Falker, S., Rounioja, S., Tschop, J., Morfeldt, E., Ferlenghi, I. \& other authors (2007). RrgA is a pilus-associated adhesin in Streptococcus pneumoniae. Mol Microbiol 66, 329-340.

Power, P. M. \& Jennings, M. P. (2003). The genetics of glycosylation in Gram-negative bacteria. FEMS Microbiol Lett 218, 211-222.

Schneewind, O., Model, P. \& Fischetti, V. A. (1992). Sorting of protein A to the staphylococcal cell wall. Cell 70, 267-281.

Schneewind, O., Mihaylova-Petkov, D. \& Model, P. (1993). Cell wall sorting signals in surface proteins of Gram-positive bacteria. EMBO J 12, 4803-4811.

Sillanpaa, J., Nallapareddy, S. R., Prakash, V. P., Qin, X., Hook, M., Weinstock, G. M. \& Murray, B. E. (2008). Identification and phenotypic characterization of a second collagen adhesin, $\mathrm{Scm}$, and genome-based identification and analysis of 13 other predicted MSCRAMMs, including four distinct pilus loci, in Enterococcus faecium. Microbiology 154, 3199-3211.

Ton-That, H. \& Schneewind, O. (2003). Assembly of pili on the surface of Corynebacterium diphtheriae. Mol Microbiol 50, 1429-1438.

van Schaik, W., Top, J., Riley, D. R., Boekhorst, J., Vrijenhoek, J. E., Schapendonk, C. M., Hendrickx, A. P., Nijman, I. J., Bonten, M. J. \& other authors (2010). Pyrosequencing-based comparative genome analysis of the nosocomial pathogen Enterococcus faecium and identification of a large transferable pathogenicity island. BMC Genomics 11, 239.

Werner, G., Coque, T. M., Hammerum, A. M., Hope, R., Hryniewicz, W., Johnson, A., Klare, I., Kristinsson, K. G., Leclercq, R. \& other authors (2008). Emergence and spread of vancomycin resistance among enterococci in Europe. Euro Surveill 13, pii: 19046.

Willems, R. J., Top, J., van Santen, M., Robinson, D. A., Coque, T. M., Baquero, F., Grundmann, H. \& Bonten, M. J. (2005). Global spread of vancomycin-resistant Enterococcus faecium from distinct nosocomial genetic complex. Emerg Infect Dis 11, 821-828.

Edited by: K. E. Weaver 